\title{
Using Three-Dimensional Printing Technology to Produce a Novel Optical Fiber Bragg Grating Pressure Sensor
}

\author{
Ying-Kai Lin, Tso-Sheng Hsieh, Liren Tsai, Shing-Hai Wang, ${ }^{1}$ and Chia-Chin Chiang ${ }^{*}$ \\ Department of Mechanical Engineering, National Kaohsiung University of Applied Sciences, \\ 415 Chien Kung Road, Kaohsiung 807, Taiwan \\ ${ }^{1}$ Department of Mechanical Engineering, Kun Shan University, \\ No. 195, Kunda Rd., Yongkang Dist., Tainan City 710, Taiwan
}

(Received August 31, 2015; accepted January 6, 2016)

Keywords: fiber Bragg grating, pressure sensor, finite element method, three-dimensional printing

In this study, an environmental acrylonitrile butadiene styrene (ABS) resin was combined with three-dimensional (3D) printing technology to develop a new pressure sensor body with the molding pressure for fiber Bragg grating (FBG) sensor technology embedded in the sensor body. The production of the new pressure sensor was completed in a hydrostatic tank, and by increasing or decreasing the pressure to simulate different FBG wavelength changes, the accuracy could be tested via finite element analysis of the experimental results. The 3D printing technology has made this process simple and has the advantages of rich variability and low cost. From the experimental results for pressure loadings of 0-4 bars, it was determined that this new FBG pressure sensor with a cylindrical configuration had a wavelength strength variation of $0.828 \mathrm{~nm}$ and a sensitivity of $0.208 \mathrm{~nm} / \mathrm{bar}$. The FBG pressure sensor with a cylindrical configuration has good linearity and reproducibility between wavelength deviation and repeat water pressure loading/unloading according to the results of a numerical analysis.

\section{Introduction}

Because of its high compressive strength, structurally engineered ceramic materials have been used for a variety of applications, such as ceramic external pressure housings for deep sea vehicles as described by Stachiw et al. ${ }^{(1)}$ Because the pressure hulls of manned submersibles are spherical, deformation is still large; (2) therefore, pressure measurement data from monitoring systems regarding changes in water pressure are an important basis for deformations and related warnings. Fiber Bragg grating (FBG) sensors can be employed in nondestructive control sector applications for structural tests, structural health monitoring, and load monitoring. ${ }^{(3)}$ In this report, we focus on fiber Bragg grating sensors, owing to the possibility that they can detect strains in specific points of complex structures and provide useful information for design and stress analysis. The fundamental concept of in-service structural health monitoring has made FBG sensors a pivotal technology in the field of real-time structural sensing. ${ }^{(4)}$ FBG sensors have been used by the American Bureau of Shipping to certify ocean-going vessels, both surface ships and submarines. ${ }^{(5)}$

Three-dimensional (3D) printing technology is based on a continuous stacking of physical layers, whereby layer upon layer of woven material is built up to generate a 3D entity. Replacing the "Corresponding author: e-mail: ccchiang@kuas.edu.tw 
traditional material processing technologies, this process is also known as additive manufacturing (AM). Currently, 3D printing technology is mainly used for prototype mold manufacturing, as well as in the fields of artistic creation, such as jewelry making, to replace processes in these fields that previously relied on traditional fine processing technology. In addition, the 3D printing technology is being introduced in bioengineering and medicine, architecture, clothing design, and other areas, and also in the innovative development of vast spaces. ${ }^{(6,7)}$ In a study by Kantaros and Karalekas, ${ }^{(8)}$ residual strains developed at the end of a fabrication process were recorded using an optical sensor with a short FBG embedded at the mid-plane of specimens built by fused deposition modeling (FDM).

This study is the first in which FBG was combined with ABS resin and 3D printing technology to produce complex cylindrical pressure sensors at a low cost. The resulting miniaturized pressure sensors eliminated the need for polymer material filling due to aging and reduced sensitivity problems associated with such fillings.

\section{Working Principle of FBG Sensors}

\subsection{Packaged FBG sensors}

In this study, FBG was used as a pressure-sensing element that was effectively embedded in the acrylonitrile butadiene styrene (ABS) resin cylinder of the sensor. The cylindrical pressure sensor scale had dimensions of $30 \mathrm{~mm}^{\mathrm{OD}} \times 30 \mathrm{~mm}^{\mathrm{H}}$ and a shell thickness of $5 \mathrm{~mm}$, while the internal components that needed to withstand the pressure had a surface thickness of $3 \mathrm{~mm}$ and a height of $20 \mathrm{~mm}$. The manufacturing procedures for the packaged FBG sensors used are shown in Fig. 1.

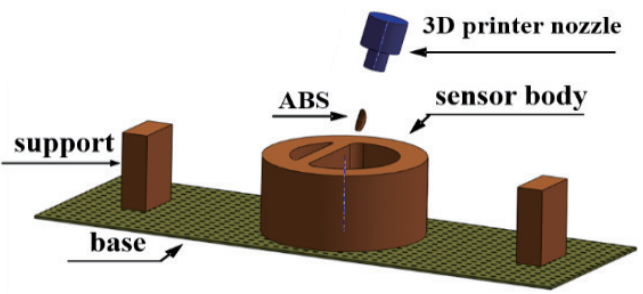

(a)

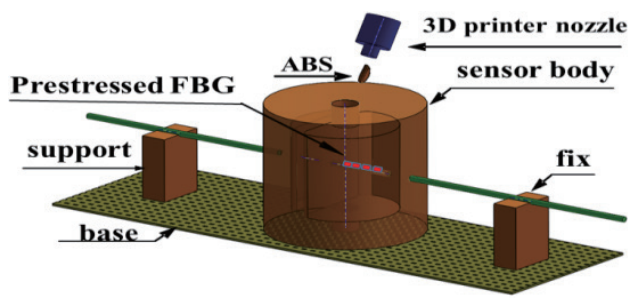

(c)

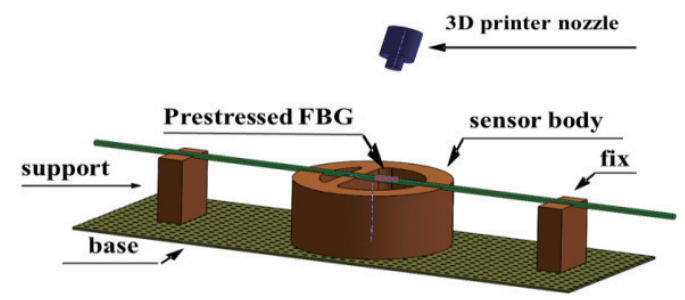

(b)

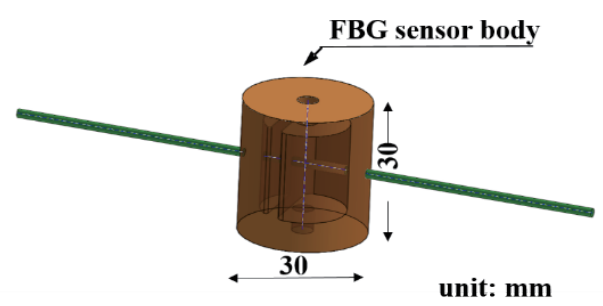

(d)

Fig. 1. (Color online) Production flow chart of the 3D printer pressure sensors. (a) Printed half of sensor body and support. (b) Prestressed FBG placed on support and fixed. (c) Printing of sensor body. (d) Removal of base and support. 


\subsection{Principle of FBG sensor operation}

FBGs rely on the measurement of the spectrum of reflected light; therefore, they may also be called reflection fiber gratings. FBGs have a grating period of around $1 \mu \mathrm{m}$. When the light emitted from a broadband light source enters the optical fiber, the light is reflected because some light is reflected and some transmitted. The light meeting the conditions of the Bragg grating is reflected, and the rest of the light is transmitted. The light rays being reflected must have a wavelength meeting the Bragg condition as expressed in the following formula: ${ }^{(9)}$

$$
\lambda_{\mathrm{B}}=2 n_{\mathrm{eff}} \Lambda .
$$

The term $\lambda_{\mathrm{B}}$ is the Bragg wavelength, $n_{\text {eff }}$ is the effective refractive index, and $\Lambda$ is the grating period. When the grating is under strain loading, the drift in the wavelength can be expressed as

$$
\frac{\Delta \lambda}{\lambda}=\left(1-P_{\mathrm{e}}\right) \varepsilon=K_{\varepsilon} \varepsilon
$$

where $\varepsilon$ is strain, $P_{\mathrm{e}}$ is the photoelastic constant, and $K_{\varepsilon}$ is the strain wavelength coefficient. According to the literature, ${ }^{(10)}\left(1-P_{\mathrm{e}}\right)$ is about 0.78 , and when the grating is subjected to tensile strain of $1 \mu \varepsilon$, the Bragg wavelength drift is approximately $0.0012 \mathrm{~nm}$.

The working principle of the sensor is that the stretching and deformation of the cylindrical strain plate installed with the FBG during changes in the liquid pressure results in shifts of the Bragg wavelength.

\section{Experiments}

The cylindrical FBG pressure sensor has been developed using 3D printing for building materials, such as ABS plastic. The FBG was packaged on the cylindrical pressure sensor, which was placed in a static water tank to undergo pressure testing at varying water pressures, as shown in Fig. 2. The experimental temperature was controlled at room temperature $\left(25^{\circ} \mathrm{C}\right)$. The optical fiber was connected to one end of the $1 \times 2$ optical coupler, and the other end was connected to a superfluorescent fiber source with a spectral analysis instrument. The FBG pressure sensor was fixed in the hydrostatic pressure tank for pressure calibration.

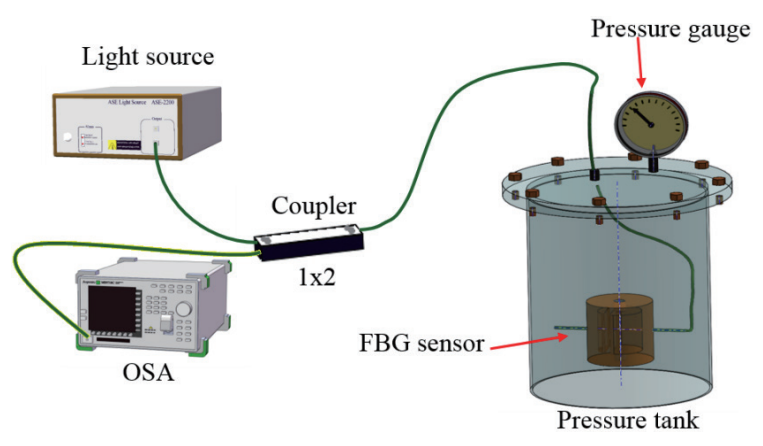

Fig. 2. (Color online) Experimental setup for the pressure-sensing test. 
The COMSOL software was then employed to analyze the strain on the pressure sensor at varying hydrostatic pressures. For experimentation, the COMSOL software was initially employed to confirm the strain values measured using the FBG for various pressures by comparing the FBG changes in the Bragg wavelength shifts as a function of liquid pressure. The results were then used to analyze the static strength of the FBG sensor under various pressure loadings.

\section{Results and Discussion}

\subsection{Analysis using the FEM}

The design of the optical fiber pressure sensor was proven to be reasonable from the experimental results. Then the finite element analysis software (COMSOL) was used for the hydrostatic test simulation, with a 3D-printed ABS material used to construct the body of the pressure sensor for combination with the FBG sensor. The properties of the ABS and optical fiber material are shown in Table 1. The element tetrahedral solid elements in COMSOL were used to construct the pressure sensor model. Six degrees of freedom were set from the nodal centers of Ux, Uy, Uz, Rx, Ry, and Rz. The FEM model had 219741 domain elements, 40480 boundary elements, and 2872 edge elements, as shown in Fig. 3. The solid strain xx in the FBG sensor under 1-4 bar loading is shown in Fig. 4.

\subsection{Hydrostatic test of the new pressure sensor}

The FBG pressure sensor with a cylindrical configuration was designed into the hydrostatic pressure sink, and the initial pressure was zero bar. The pressure was increased at 0.5 bar intervals up to 4.0 bars. Additional pressure was not added until the pressure from the previous addition of 0.5 bar stabilized. The wavelength at zero bar was $1551.06 \mathrm{~nm}$, and that at 4.0 bar was 1551.88 $\mathrm{nm}$. Figure 5 shows the drift in the wavelength as the cylindrical sensor was compressed. The

Table 1

ABS and optical fiber material properties.

\begin{tabular}{lccc}
\hline Material & Elastic modulus $(\mathrm{MPa})$ & Poisson's ratio & Density $\left(\mathrm{kg} / \mathrm{m}^{3}\right)$ \\
\hline ABS & 120 & 0.275 & 850 \\
FBG & 73000 & 0.165 & $2.2 \times 10^{6}$ \\
\hline
\end{tabular}

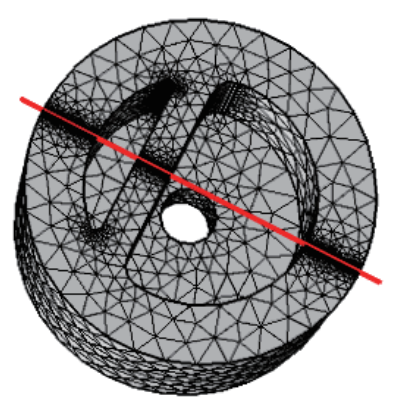

Fig. 3. (Color online) FEM model mesh elements for the pressure sensor body. 

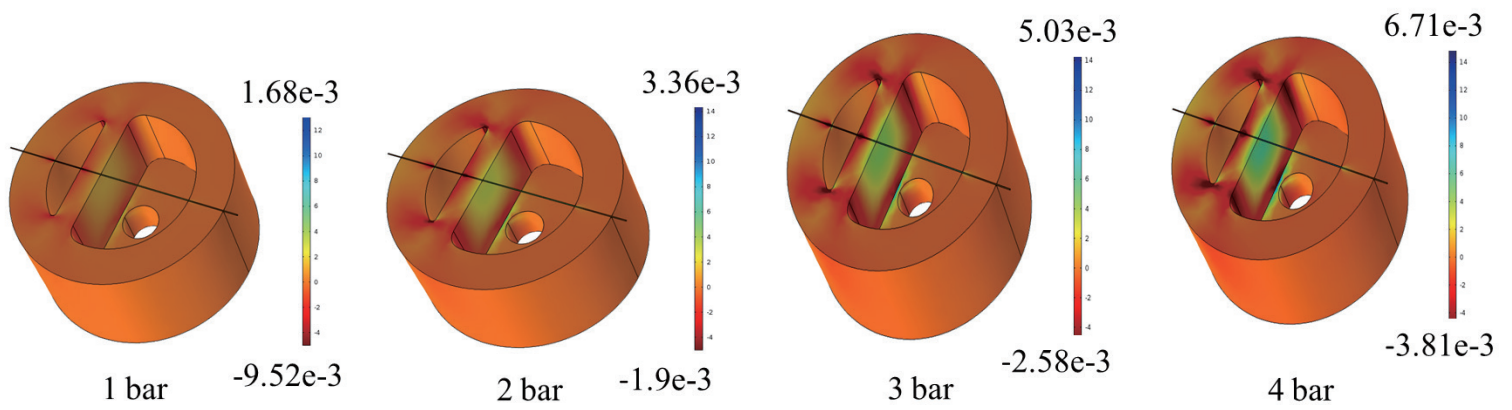

Fig. 4. (Color online) Strain of the FBG sensor under $1-4$ bar loading.

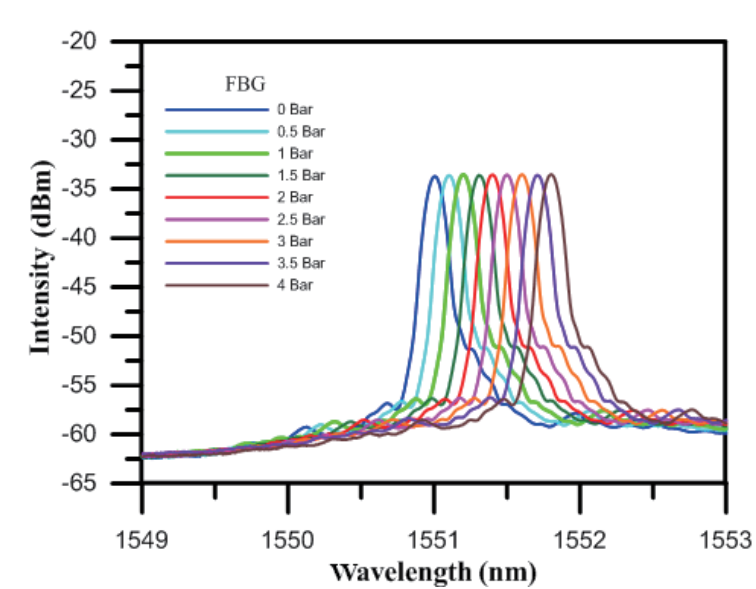

Fig. 5. (Color online) Spectrum of the FBG pressure sensor under various loadings.

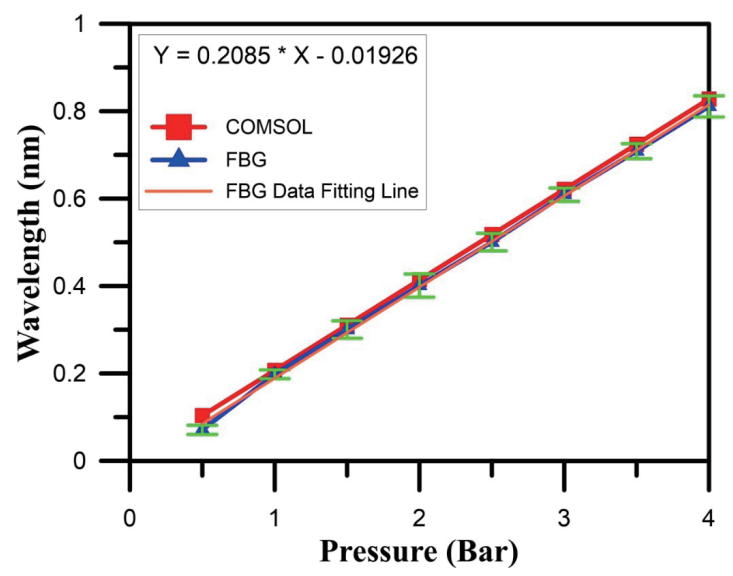

Fig. 6. (Color online) Comparison of the simulated and experimental results for the FBG pressure sensor.

wavelength drift $\Delta \lambda$ increased by approximately $0.208 \mathrm{~nm} / \mathrm{bar}$. The values measured using the cylindrical FBG pressure sensor for loadings from 0 to 4 bars were verified by experimental measurement. When the pressure was 4 bars, the strain in the wavelength strength variation was 0.828 $\mathrm{nm}$; the sensitivity of this pressure sensor was $0.208 \mathrm{~nm} / \mathrm{bar}$, and strain variation in the COMSOL software simulation analysis was also proportional to the pressure loading, as shown in Fig. 6.

\section{Conclusions}

In this study, a FBG was used as the pressure-sensing element in combination with 3D printing technology, resulting in the successful production of a new type of pressure sensor. From a static water pressure experiment, we found a linear relationship between the wavelength of the FBG sensor and the pressure loading when there was deformation of the solid material of the pressure sensor due to loading from 0 to 4 bars. The sensitivity of the cylindrical pressure sensor was about $0.208 \mathrm{~nm} / \mathrm{bar}$. Because the ring stiffeners of the shell are very strong and the deformation spacing between them is smaller than the interior strain plate of the cylindrical pressure sensor, the Bragg wavelengths experience a large shift. 


\section{Acknowledgements}

This work was supported by the Ministry of Science and Technology, Taiwan (grant number MOST 103-2221-E-151-009-MY3).

\section{References}

1 J. D. Stachiw, D. Peters, and G. McDonald: Ceramic External Pressure Housings for Deep Sea Vehicles (Oceans Publishing, Boston, 2006) pp. 1-7.

2 Y. Yano and S. Takagawa: MTTS/IEEE Techno-Oceans 3 (2004) 1554.

3 M. Iodice, V. Striano, G. Cappuccino, A. Palumbo, and G. Cocorullo: Proc. Fibres and Optical Passive Components (2005) p. 307.

4 Y. C. Chena, C. C. Hsiehb, and C. C. Lina: Sens. Actuators, A 167 (2011) 63.

5 J. S. Kiddy, C. S. Baldwin, and T. J. Salter: Proc. MTS/IEEE Oceans 2 (2005) 1876.

6 K. Pataky and J. Brugger: Rapid Prototyping of Biomaterials, ed. R Narayan (Woodhead Publishing, 2014) pp. 75-96.

7 D. Dimitrov, K. Schreve, and N. de Beer: Rapid Prototyping J. 12 (2006)136.

8 A. Kantaros and D. Karalekas: Mater. Design 50 (2013) 44.

9 M. Majumder, T. K. Gangopadhyay, A. K. Chakraborty, K. Dasgupta, and D. K. Bhattacharya: Sens. Actuators, A 147 (2008) 150.

10 R. Kashyap: Principles of Optical Fiber Grating Sensors (Academic Press, Boston, 2009) 2nd ed., Chap. 10, pp. 441-502. 